Borneo Journal of Sciences and Technology, Volume (1), Issue (2), Pages: 51-61

DOI: https://doi.org/10.35370/bjost.2019.1.2-08

e-ISSN: 2672-7439

(C) 2019, UCTS Publisher.

Submitted: $9^{\text {th }}$ April 2019

Accepted: $30^{\text {th }}$ April 2019

Published: $31^{\text {st }}$ July 2019

\title{
Existing Practices in the Evaluation and Selection of e-Learning Software: A Survey
}

\author{
${ }^{1}$ Ahmad Fadli Saad, ${ }^{2}$ Liyana Shuib \\ ${ }^{1}$ School of Computing and Creative Media, University of Technology Sarawak, No 1, Jalan Universiti, \\ Sibu Sarawak, Malaysia \\ ${ }^{2}$ Department of Information Systems, Faculty of Computer Science and Information Technology, \\ University of Malaya, Kuala Lumpur and 50603, Kuala Lumpur, Malaysia
}

\begin{abstract}
This study aims to report a study on organization practices related to the process of existing evaluation and selection of e-Learning software. Initially, questionnaires were distributed to 250 experts who had been contacted via email or through walk-ins. However, only fifty experts which were categorized into decision makers, academicians/researchers and technical experts participated to fill the questionnaire. The results obtained from this study showed that the e-Learning software evaluation is a complex task as it requires many processes, evaluation criteria, and suitable evaluation techniques. The existing software evaluation criteria are insufficient to evaluate various e-Learning software products. This is due to the general characteristic of the criteria that can be used to evaluate any software. The outcome of this study provides organizations and e-Learning practitioners with information on existing practices when organization evaluates and select e-Learning software in their organizations. It can be used as a guideline for their e-Learning planning and implementation by organization Not least, it also gives the opportunity for the academicians/researchers to undertake a future study based on the existing e-Learning software evaluation and selection problems obtained.
\end{abstract}

Keywords: Existing Practices, e-Learning Software, Evaluation and selection of e-Learning Software.

\section{INTRODUCTION}

The current proliferation of e-Learning has prompted the emergence of various e-Learning software (e-LS) that support the implementation of e-Learning in organizations. The size of the eLearning market was valued to be more than USD 165 Billion in 2015 and is likely to grow by 5\% between 2016 and 2023, exceeding USD 240 Billion [13]. There has been an increasing interest in the implementation of e-learning in Universities, colleges as well as private institutions of higher educations and other organizations in Malaysia [18]. Industries in Malaysia also adopting elearning to enhance the operational effectiveness of the organization [2]. National online learning portal, The Malaysia Education Online (MEdO) with the objective of expanding international distance learning was launched in April 2011. One of the goals of the e-Learning policy in Malaysia is to have no less than $30 \%$ of all higher education institution courses conducted on-line by 2015 [1]. Today many Universities in Malaysia have implemented eLearning [18]. Sree Knowledge Provider (SKP), a private organization has implemented fifteen degree programs via online to offer e-Learning opportunities for business management in Malaysia [1]. The demand for e-Learning has motivated vendors or developers to produce e-LS for the implementation of e-Learning in organizations. Furthermore, the procurement of e-LS is highly expensive as it consumes a significant portion of the capital budget of an organization. E-LS cost thousands and even millions of dollars. The organizations have to make the correct choice of e-LS. The selection of wrong e-LS will cause the organization to lose a lot of money. At the moment, there is not much information available as a guideline on the evaluation and selection of e-LS for e-Learning implementation. Thus making selection decision for the procurement of suitable e-LS is difficult. Therefore, it has motivated this study to conduct an investigation on the existing practice among organization when they evaluate and select e-LS.

\section{RELATED WORKS}

E-learning is considered as the latest approaches of bringing educational materials in higher education institutions around the world [4]. E-Learning is associated with Advanced Learning Technologies 
(ALT), which deals with technologies and methodologies in learning using networking or multimedia technologies [16]. E-Learning gives users freedom in following a preferred educational path, together with control to explore effective paths. This is beneficial for the students, resulting in a deeper understanding of the instructional material [15]. Education can take place anywhere as long as there are computers and an Internet connection available in homes, workplaces and Internet cafes [5]. E-Learning can be useful for organizations, including industries and academia, and has been adopted as an alternative training method to improve efficiency, and for staff training through the use of interactive learning [7]. eLS can be categorised as Learning Management Systems (LMS), Learning Contents Management Systems (LCMS) [22-23]. Besides, deployment tools like Java technology are also used to develop e-Learning [14]. e-LS provide support for customising and developing e-Learning applications [27-28]. With the verities of e-LS, different e-LS having different criteria to support the implementation of e-Learning. Thus, is difficult for organizations to select suitable e-LS.

In general, there are several stages as a guideline in the software evaluation and selection as described in [19-20]. The stages are requirements definition; preliminary investigation; short listing of packages; establishing the evaluation criteria for the evaluation; evaluating the software package; selecting the software package; negotiating with the software vendor; buying and implementing of the majority appropriate software package. Like other software evaluation, the e-LS evaluation and selection also involve numerous stakeholders, stages, evaluation criteria, e-LS alternatives, evaluation methods, and technique. Thus, the evaluation and selection processes for the procurement of e-LS are complex, time consuming and costly. Some studies have conducted the evaluation of different types of e-LS. García (2006) developed a framework to evaluate the e-Learning platform by using the SCORM standard specifications [17]. The framework lets instructors using benchmark tests to evaluate e-learning platforms. The utilisation of the Analytical Hierarchy Process (AHP) technique was proposed to evaluate e-learning systems from the perspective of user satisfaction [28]. Colace \& De Santo (2008) proposed a model to evaluate commercial e-Learning platform [24]. Padayachee et al. (2011) proposed the utilization of the ISO 9126-1 quality model for the user in the selection of generic external systems quality criteria and sub-criteria for the evaluation of Content Management Systems (CMS). These studies have focused on the evaluation of the specific study of e-LS products using a specific technique. As many e-LS are continuously produced in the market, a study in obtaining the latest practice in the
e-LS evaluation and selection is required. Therefore, this study aims to answer the following research questions:

RQ1: How do organizations implement the e-LS?

RQ2: What are the stages, methods and evaluation techniques in the e-LS evaluation and selection?

RQ3: How do organizations identify the e-LS evaluation criteria?

RQ4: What are the support tools used to help organization in the e-LS evaluation and selection?

RQ5: What are the problems that organization are faced in the e-LS evaluation and selection?

This study is organised as follows: Section 2 review related works of the study. Section 3 explains the research methodology; Section 4 presents the results and discussion from the survey, Section 5 gives the recommendations, while Section 6 concludes the study

\section{METHODOLOGY}

This section describes the research method used in the study.

\section{Survey}

A survey is used in this study. Using survey is acknowledged to be an acceptable research tool to gain a reflection of the attitudes, preferences and opinions of a community [25]. Thus it was used as a suitable research tool to collect data in this study. The instrument used to collect data on the evaluation and selection of e-LS is questionnaire. The information collected for this study were the experts' background, e-LS implementation, the evaluation and selection stages and method of e-LS, e-LS evaluation criteria, support tools utilized in the eLS evaluation, and problems faced in the evaluation process.

\section{Questionnaire Design}

The questionnaire is divided into two sections. The first section consist of demographic information about the experts. The second section, require the experts to answer questions relevant to the existing practices of organizations in the evaluation and selection of e-LS. The questions in the questionnaire undergo_several revisions by the experts. The questionnaire was reviewed in a pilot study involving three experts in e-LS 
to establish the clarity of the questions. As to improve the content of the questionnaire, some modifications on its questions were then made.

\section{Experts}

Any individual having relevant knowledge and experience of a particular topic is considered an expert [30]. In the survey, the experts are the Decision Makers and Technical Experts. Besides, Academicians/Researchers that conduct research in eLearning are invited. They came from the organization that implemented e-Learning and had knowledge of eLearning and e-LS. Some of the academicians/researchers were appointed by their universities as the Director or e-Learning Coordinator in e-Learning unit. They were responsible for the e-LS evaluation and selection. Some of them were introduced directly by the respective staff when conducting the survey at their organization. Initially, 250 experts were contacted via phone calls or emails. Appointments were made to meet experts face-to-face for those who were contacted by phone. For those who were invited through email, they were required to answer an online questionnaire. Out of the 250 experts contacted, only 53 of them participated in the survey, of which 50 experts completed the questionnaire and 3 experts did not.

\section{Conducting the Survey}

The information collected included demographic information about the experts' background. Pertinent questions on current practices with regard to the evaluation and selection of e-Learning software in organizations were also asked.

\section{Data Analysis}

The data analysis was based on the respond given by the 50 experts in the questionnaire. Descriptive statistics were employed to analyse the results of the survey. The analysis of the descriptive statistics was supported by the well-known statistical software, the Statistical Package for Social Science (SPSS) version 15.0.

\section{RESULTS}

This section discusses the findings obtained from this study. The results were divided into two parts: demographic background, and current practices in the eLS evaluation and selection.

\section{Demographic Background}

The demographic background served to verify the experts' qualifications. The demography include information about the experts' type of organizations, job description, educational attainment and years of working experience. The experts were from 8 public universities, 2 private Universities, 5 private organizations and 1 Government Agency that implement e-Learning in Malaysia. $78 \%$ of the experts were university staffs; $10 \%$ were serving in the semigovernment; $6 \%$ were the private sector, and $6 \%$ were working in software companies. Majority of the experts are from educational institutions. They were categorised based on their job function. Of all of the experts, $24 \%$ were decision makers and $40 \%$ were academic practitioners including lecturers and researchers. The remaining 36\% were technical experts who were directly involved in the e-Learning implementation. The survey result discovered that $26 \%$ of the experts had a $\mathrm{PhD} ; 44 \%$ were Master's degree holders; 24\%, were Bachelor's degree holders; and 6\% were diploma holders. Moreover, as for the expert's working experience, the result found out that $8 \%$ had 0 5 years of experience; $30 \%$ had 6-10 years of experience; $46 \%$ had $11-15$ years of experience; $8 \%$ had 16-20 years of experience, and $8 \%$ had above 20 years of experience.

\section{Existing practices on e-LS evaluation and selection}

When seeking the information on the purpose of organization using e-LS as demonstrated in Figure 1, $38 \%$ out of the experts used e-LS for in-house e-Learning application development; $36 \%$ use e-LS for research or academic purposes; $24 \%$ use e-LS to develop e-Learning applications for the purpose of business, while $14 \%$ customized ready-made e-LS.

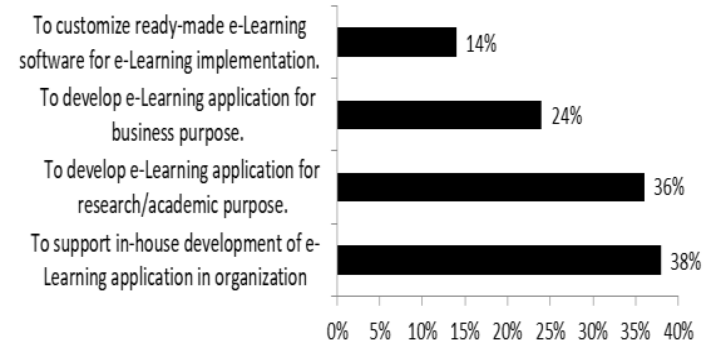

Figure 1: The purpose of using e-LS

With regards to the approaches that the organizations implement e-LS as shown in Figure 2, $44 \%$ of the experts developed e-Learning applications in-house; $32 \%$ of the experts implemented e-Learning by cooperating with outside vendors; $28 \%$ of them purchased off-the-shelf or ready-made software products; and the remaining $4 \%$ direct purchased e-Learning applications from vendors. 


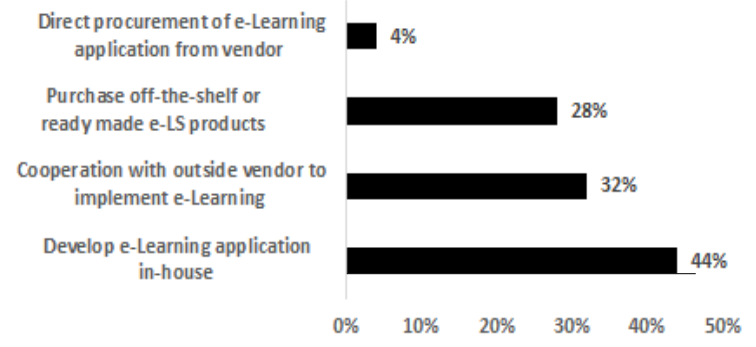

Figure 2: The approach that the organizations used to implement e-LS

In the e-LS implementation both open source and commercial software were used as illustrated in Figure 3(a) and Figure 3(b). Moodle is the most popular open source of e-LS. $72 \%$ of the experts used Moodle to implement e-Learning. The remaining $22 \%$ of the experts used Joomla, 4\% used Oracle i-Learning, and only $2 \%$ of the experts used Fle 3 . When probed about commercial e-Learning software, out of $50,6 \%$ of the experts specified that they used WebCT, $4 \%$ noted that they used Web Learning LMS, $4 \%$ indicated that they used Blackboard Learn and 2\% cited that they used Lotus Learning Spaces. Others used other commercial eLS providers such as, i3-learning solutions $(2 \%)$ and Claroline (2\%).

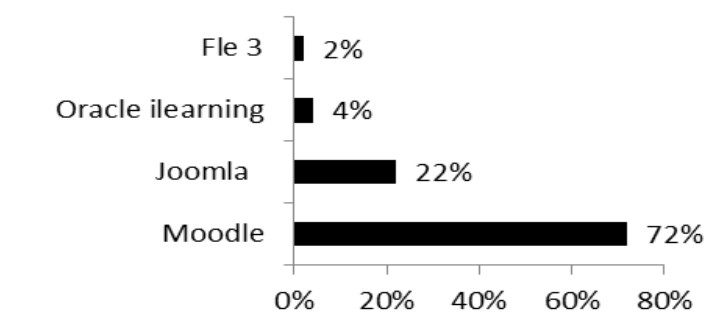

Figure 3(a): Open Source e-LS

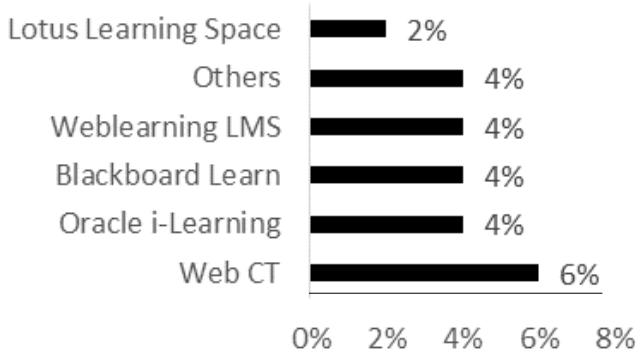

Figure 3(b) Commercial type of e-LS

As in the use of type of operating system used for e-LS as shown in Figure 4, the most popular was Linux at $56 \%$ and Windows was the second popular OS used at $44 \%$.

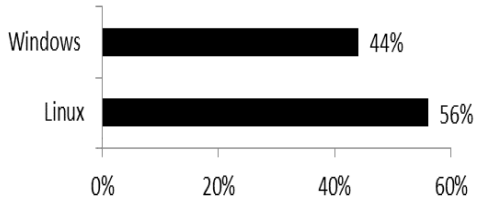

Figure 4: Operating Systems for e-LS

In determining the type of Database Management Systems (DBMS) used with the eLearning application as can be seen in Figure 5, most of the experts or $76 \%$ of them used MySQL, 20\% used SQL Server, while only $4 \%$ used Oracle. This showed that the open source of DBMS is more popular than commercial DBMS for e-Learning implementation among organizations in Malaysia.

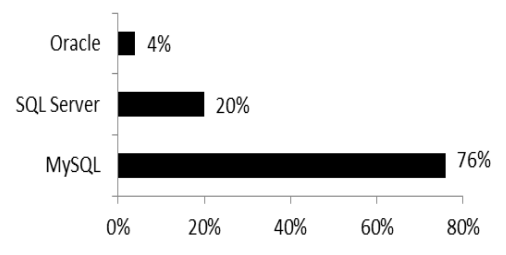

Figure 5: DBMS for e-LS

In identifying the programming languages used for e-LS, as presented in Figure 6, the survey indicate that the majority of the experts, i.e. $94 \%$, used PHP, 20\% used Java Script, and 4\% of the experts used other programming languages (ASP.Net and Java programming).

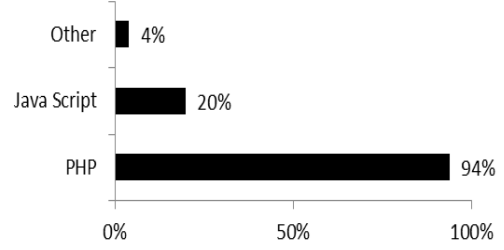

Figure 6: Programming languages used for e-LS

In seeking the types of web server used in the implementation of e-LS, this study found out that $82 \%$ of the experts used Apache, 14\% used Microsoft IIS, and $4 \%$ of the experts used another web server, namely Apache Tomcat as displayed in Figure 7. 


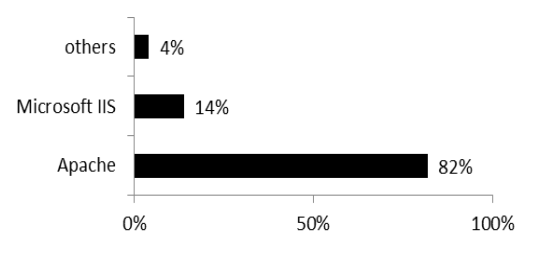

Figure 7: Types of Web Servers

The study also asked the experts to note the people participated in the evaluation and selection process as illustrated in Figure 8. Majority of them are technical experts at $76 \%$, followed by those in top management at $68 \%$, and $14 \%$ were software vendors/suppliers. $8 \%$ of the groups were the end users. This result indicate that most organizations involve their top management and technical experts, while a few organizations invite software vendors/suppliers and end users into the evaluation and selection process of e-LS.

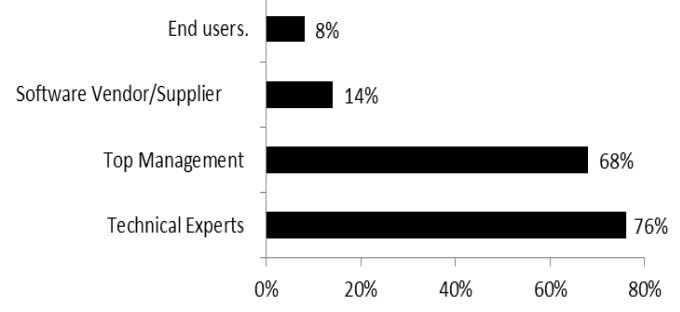

Figure 8: People participated in the evaluation and selection process of e-LS

This study also identified the stages that the experts applied in the evaluation and selection of e-LS as shown in Figure 9. The study revealed that $84 \%$ of the experts indicate that they applied the preliminary investigation stage; $68 \%$ of them opted the requirements definition stage; $68 \%$ of the experts selected for the shortlisting of packages; $60 \%$ of them indicated evaluating the software package stage; $58 \%$ of the experts selected the software package; $52 \%$ of the experts established the evaluation criteria for the evaluation stage; $36 \%$ of the experts applied the purchasing and implementing of the most appropriate software package stage; and $22 \%$ of the experts realized the negotiating with the software vendor stage.

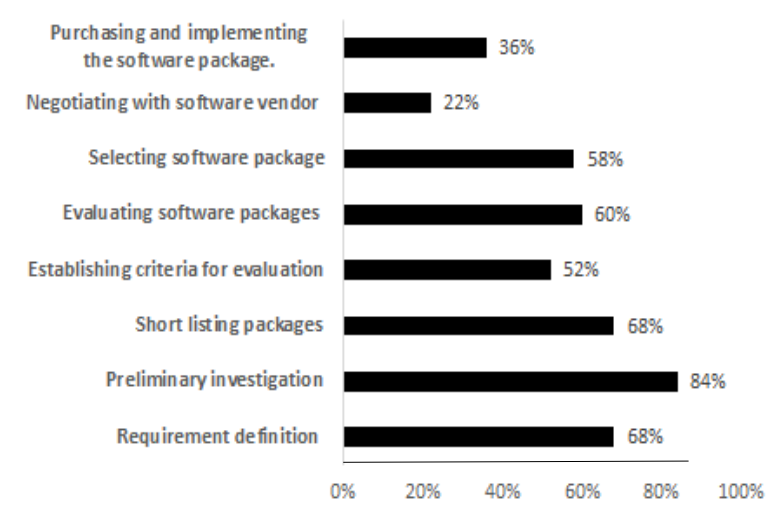

Figure 9: The stages applied in the evaluation and selection of e-LS

The survey also discovered how the organizations identified the e-LS evaluation criteria which would be incorporated in the evaluation and selection process as demonstrated in Figure 10. 64\% of the experts rely on internal meetings and product brainstorming; $44 \%$ of them got the information through ad-hoc meetings; $42 \%$ via website/Internet surfing; $30 \%$ referred to end users; $28 \%$ of the experts asked vendors to provide proposals; $28 \%$ contacted and interviewed users; while the $20 \%$ referred to pamphlets, catalogues, articles, products and documentations.

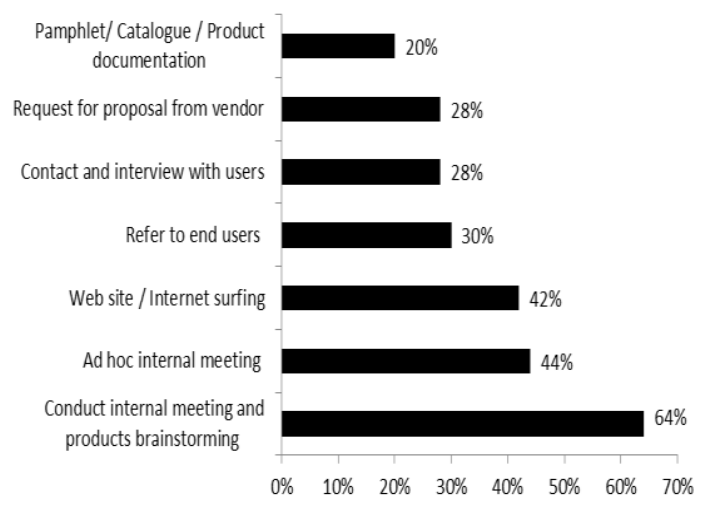

Figure 10: How organization identified the e-LS evaluation criteria

As for the methods and techniques used for the evaluation and selection of e-LS as highlighted in Figure 11, 68\% of the experts noticed that they conducted internal meetings; $66 \%$ through reviews of documentations, pamphlets and articles from software providers; $28 \%$ select the weight method; $24 \%$ made opt for the benchmark reports; $18 \%$ applied the rank technique; $16 \%$ chose the scoring method; and only $4 \%$ selected the Analytical Hierarchy Process (AHP) as their evaluation technique. There were no experts applied other evaluation techniques, such as Fuzzy AHP. 


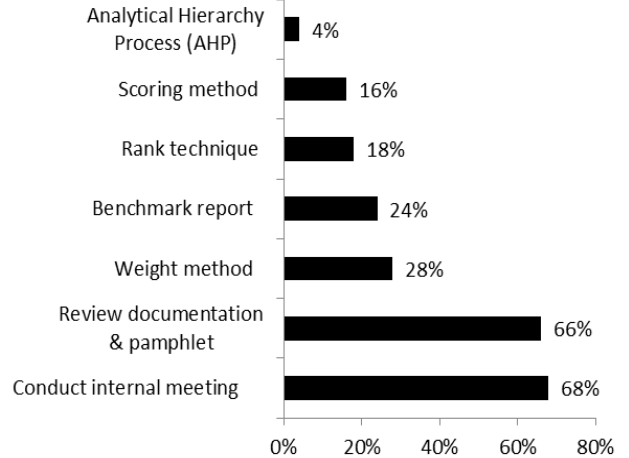

Figure 11: Methods and techniques used for the evaluation and selection of e-LS

In defining how the organizations determined the evaluation criteria for e-LS as depicted in Figure 12, $54 \%$ of the experts surfed websites and the Internet; $30 \%$ relied on the evaluation criteria provided or suggested by vendors; $22 \%$ conducted internal meetings and brainstorming; $20 \%$ of the experts contacted and interviewed users; and 18\% referred to pamphlets/catalogues/articles and product documentations. Meanwhile, only $6 \%$ of these experts referred to the ISO/IEC 9126-1 quality model to determine the evaluation criteria for e-LS.

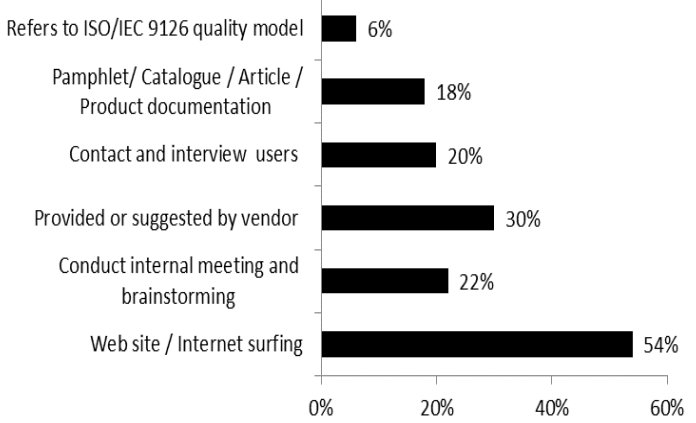

Figure 12: How organizations determined the evaluation criteria for e-LS

The experts were also asked to specify the evaluation criteria when selecting e-LS as presented in Figure 13. Based on the experts' responds, the criteria that the organizations took into consideration when selecting software were: $94 \%$ cost; $90 \%$ functionality; $76 \%$ maintainability; $78 \%$ usability; $74 \%$ reliability; $60 \%$ efficiency, 56\% vendor/supplier; 54\% product benefits; $52 \%$ portability; $42 \%$ risk and uncertainty, and $34 \%$ the organizational aspect. The high criterion that was considered by the organizations in the selection of e-LS is cost, while the least considered was the organizational aspect.

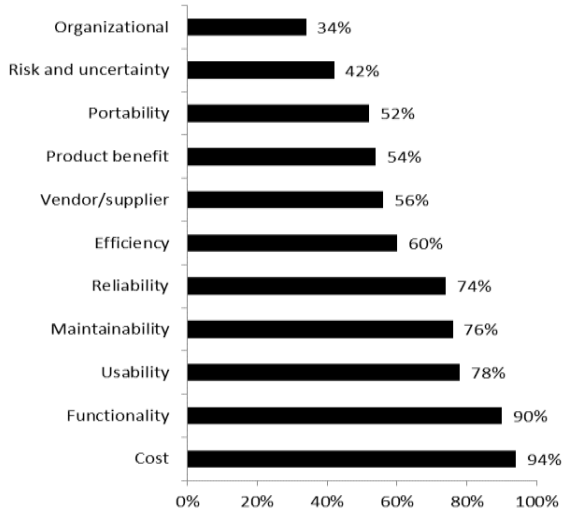

Figure 13: The evaluation criteria that organization considered in the evaluation process

As potrayed in Figure 14, the experts were also asked whether the organizations used any support tools to assist in the evaluation of e-LS. The survey revealed that only $14 \%$ of the experts indicate their organizations used support tools, while $86 \%$ of the experts showed that their organizations did not use support tools. Out of $14 \%$ or 7 of the experts who used support tools, 6 experts used a spreadsheet (e.g. Microsoft Excel, Lotus Notes), while 1 expert used an application software for decision making (Expert Choice).

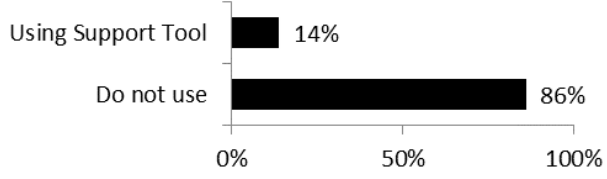

Figure 14: The use of tools to assist in the evaluation process

In viewing the effectiveness of software tools, as shown in Figure 15, those 7 of the experts who used support tools were asked to answer questions on the effectiveness of using the support tools. It was found out that only 1 out of 7 experts who used support tools answered that the support tools are effective, meanwhile 6 of the experts stated that they are not.

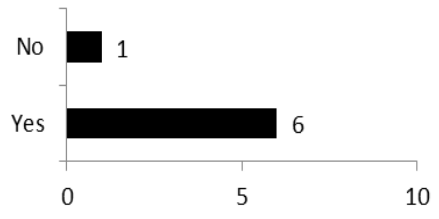

Figure 15: The effectiveness of support tools 
Among the experts who noticed that the software is not effective, 4 of the experts thought the software did not provide enough information about the e-LS evaluation criteria and the software metrics for decision making, 3 of the experts showed that it was difficult to use, and 2 of the experts claimed that the software did not provide a technique for measuring uncertain characteristics in the evaluation criteria as described in Figure 16.

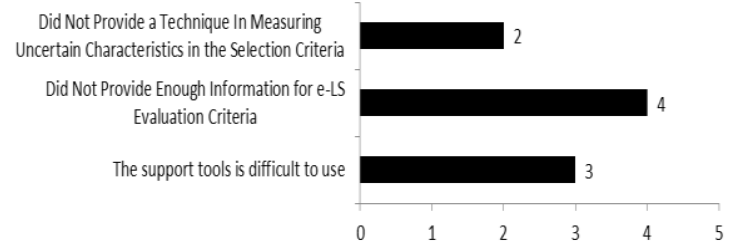

Figure 16: Experts provide information on why the support tool was not effective

The experts were also asked whether the support tools could assist in the process of evaluating and selecting e-LS as can be seen in Figure 17. The majority of the experts, i.e. $92 \%$, indicated that the support tools could assist in the process, while only $8 \%$ of the experts thought otherwise.

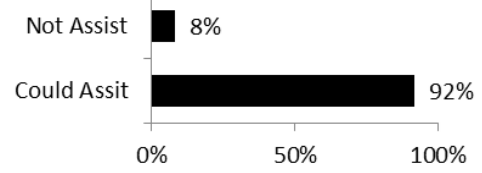

Figure 17: Experts' opinion on whether support tools assisted in the evaluation process

This study also require the experts to indicate if their organization faced any problems in the evaluation and selection process of e-LS as listed in Figure 18.

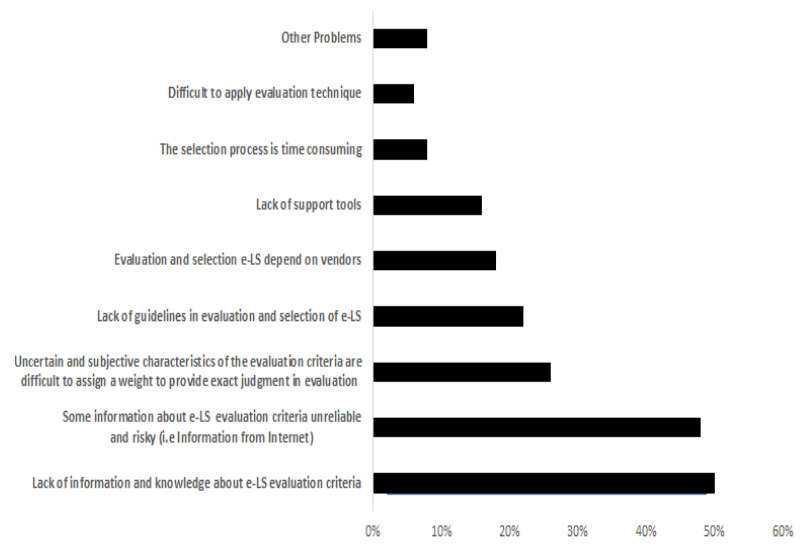

Figure 18: Problems faced in the evaluation and selection process of e-LS

Out of all of the experts, $50 \%$ said their organization encountered problems due to lack of information and knowledge about e-LS evaluation criteria; $48 \%$ of them indicate that some information about the e-LS evaluation criteria were unreliable and are risky, such as information obtained from the Internet; $26 \%$ of the experts responded uncertain and subjective characteristics of the evaluation criteria, which made it difficult to assign a weight or to provide an exact judgment in the evaluation; $22 \%$ of the experts noted the lack of guidelines in the evaluation and selection of e-LS, $18 \%$ of the experts thought that vendor dependency is one of the problems; $16 \%$ indicate a lack of support tools; $8 \%$ opined that the selection process was time consuming; $6 \%$ indicated the problems were due to difficulties in applying the evaluation technique; and $8 \%$ of the experts highlighted other problems.

The discussion of this study answered the four RQs as follows:

RQ1: The e-LS implementation

All experts of this study came from organizations that were using e-LS to implement e-Learning. Mostly, the e-LS products were used to support the in-house development of e-Learning applications in organizations. The e-LS also were utilized for academic or research purposes. Less than half of these organizations were using e-LS to develop e-Learning applications for business purposes or for customizing ready-made e-LS for the implementation of e-Learning. This shows that organizations choose to develop their own e-Learning applications compared to purchasing the ready-made software. As to support the in-house development of e-LS applications, mostly, Malaysian organizations use open source-based e-LS. Some of the experts were using Java technology, which enables users to construct e-Learning applications from scratch, 
without depending on LMS and CMS features. The study revealed that the most used open-source LMS is Moodle. Typically, Moodle is used together with Linux OS, MySQL, PHP and Apache as the web server. A few organizations use commercial products due to the cost concern. Organizations prefer open source rather than commercial e-LS. Moreover Open Source provides flexibility to develop an e-LS application instead of being bound to the criteria offered by commercial products. The staff involves in the evaluation and selection of e-LS were technical experts and members of the management. They were evaluated suitable e-LS based on their knowledge and experience. This will ensure that the organization procures the correct choice of e-LS. RQ2: Stages, methods and techniques in e-LS evaluation and selection.

RQ2: Stages, methods and techniques in e-LS evaluation and selection

The whole evaluation process can be tedious and complex, with many stages and activities that need to be carried for the procurement of e-LS. Some stages required the utilization of suitable methods and technique in the evaluation process. Moreover, these stages or their activities are general in nature and act only as a guide for software evaluation. This survey also determined the stages that organizations adhere to in the evaluation and selection of e-LS. Most of the experts selected the preliminary investigation stage, which indicated that they went through the stage. The next option indicated that they established the criteria before the evaluation. The negotiation with the vendor stage was mostly ignored by the experts, as only a few of them carried out this stage in their evaluation of e-LS. The remaining experts skipped this stage altogether. This stage seemed to be the least important among all the stages to be conducted in the whole evaluation process. Only half of the experts noted that they determined the criteria before the evaluation by using a specific technique, while the remaining stated that they evaluated the software, particularly by rating the criteria against each other and the software products. These two stages can be considered to be essential, and it is expected that all experts would have implemented these stages in some form or another. There was an inconsistency in the implementation of these stages. All or some of these stages might need to be further defined for the e-LS evaluation. Most organization in Malaysian do not strictly adhere to all the stages when evaluating and selecting e-LS. Very few organizations delivered all the stages. Other organizations skipped or did not carry out one or two stages. This means a few experts did not implement or go through all the stages completely. They skipped a few of these six stages. They could have conducted it differently from the way it was defined in the questionnaire. Cost, time and the complexity of carrying out each and every one of the stages may have been factors as to why some organizations did not undergo all the stages. It is unclear how this affected the evaluation and selection outcomes. The organizations preferred brainstorming sessions, ad hoc internal meetings, product websites, vendors, end users and pamphlets to identify which e-LS was to be included in the evaluation process. Obtaining information on the e-LS products via those means is risky since the information might be incomplete, incorrect and biased. An important step in the software products selection is the technique used in the evaluation process. The technique that is commonly used for software evaluation is AHP [12][21][29] [26][33]. The AHP technique used in the evaluation of e-LS has also been reported in [10]. Besides, benchmarking is also used as a technique in the evaluation of e-LS [8][17]. The Malaysian organizations, however, preferred to evaluate e-LS via internal meetings, documentation reviews, weight method and benchmark reports. A few of them used AHP and other techniques. AHP-based techniques can be complicated if done manually, which probably explains why these techniques are not common among Malaysian organizations. Finding from this study found that none of the organizations used the Fuzzy-AHP.

\section{$R Q 3:$ Identification of the e-LS evaluation criteria}

Determining and establishing the criteria to be used for the evaluation is one of the important evaluation and selection stages of the software products. There are three main sources of information by which the organizations determined the evaluation criteria for e-LS were the Internet, internal meetings and vendors. The evaluation criteria of software products such as e-LS can also be found in the ISO/IEC 9126-1 quality model. It is a standard quality model that is commonly used to determine the criteria to evaluate software products [14]. It provides the criteria to be considered for the evaluation of software products, such as functionality, usability, maintainability, reliability, efficiency and portability [31]. Yet very few experts referred to this quality model when establishing the criteria to evaluate e-LS. This could be attributed to the unique content of the e-LS when comparing with the other software products. The criteria provided in this quality model may be inadequate for e-LS evaluation. The cost criterion, which is not a quality factor criterion, was the most included or evaluated criterion. This was followed by a few criteria from the quality factor such as functionality, usability, maintainability, reliability and efficiency that can be found in the ISO/IEC 9126-1 quality model. Besides cost, they also considered other criteria such as vendor, product benefits, risk and uncertainty as well as organization. 
RQ4: Software/support tools used to help in the e-LS evaluation and selection

This study also searched any software or support tools that could be useful in the evaluation stage, where evaluation techniques were in the e-LS evaluation. It found that many experts did not use software or support tools in the evaluation and selection process. That could be due to the simple evaluation technique were comprised of very minimal mathematical applications. For example, the weight score method and ranking method are straightforward, where a weight or rating scale is assigned to each criterion [32]. Since these methods do not require complex calculations, a simple tools like a spreadsheet could be used to analyse the evaluation data. Thus in this study, only a few experts made use of software tools to help in the evaluation and selection process. Those who used support tools mostly used a spreadsheet software tool such as Microsoft Excel. The study found that the techniques that they used were not mathematically complicated. It could explain why a general-purpose spreadsheet like Excel was adequate. In addition, for those who used other techniques, the AHP technique and a generic decision making tool like Expert Choice [11] was available in the marketplace to assist them in analyzing the data. Yet, many of those who used software tools did not think the tools were effective in assisting them due to usability issues, inadequate evaluation criteria and metrics, and unsuitable techniques to address uncertainty in the evaluation. Majority of the experts believed that the support tools could assist in the evaluation and selection process. This was not surprising considering the different and many stages in the evaluation and selection process, where a support tool in each or most of the stages could be beneficial.

RQ5: Problems faced in the e-LS evaluation and selection

This study found that in Malaysia, the top three problems that the organizations faced in evaluating e-LS were interrelated to the evaluation criteria. The organizations were not sure if the criteria that they obtained from sources like the Internet, vendors or internal meetings were relevant, trustworthy or adequate for the e-LS evaluation. They also specified the uncertainty in the evaluation of some criteria. For example, it was difficult to assign exact numbers as weights to the evaluation criteria in the judgment. Many of the investigated organizations relied on the Internet and other sources to identify the evaluation criteria as there was no specific standard quality model for the e-LS evaluation. The evaluation criteria provided by the ISO/IEC 9126-1 quality model are relevant and adequate for evaluating general software products. However, software for a specific purpose, such as e-LS, the criteria in the ISO/IEC 9126-1 quality model may not be enough or comprehensive. The elements related to learning in e-LS should also be considered in the evaluation. However, the ISO/IEC 9126-1 quality model does not include the criteria related to such elements in e-LS evaluation. Other problems encountered in the evaluation and selection process involved a lack of guidelines to evaluate e-LS, lack of support tools, the time consuming selection process, and difficulty in applying the evaluation technique. The common evaluation and selection stages can be used as a guideline for any software evaluation. However, determining the evaluation criteria stages did not provide any information on the suitable e-LS evaluation criteria. In addition, the evaluation stage also did not provide any information on suitable evaluation techniques in the e-LS evaluation. Cconsidering the number of different stages that decision makers have to go through in evaluating any software, the evaluation and selection process is time consuming. Evaluation techniques, such as weight scoring, can be tedious if done manually, and those with complicated mathematical underpinnings can be difficult to apply. This could justify why the experts stated one of the problems is a lack of software tools available to assist in the e-LS evaluation and selection process. The existing results also found out that the experts thought that a support tool could assist them in the e-LS evaluation process. Other than the problems stated in the questionnaire, the experts also provided other problems that they were faced when evaluating e-LS. The e-LS being unable to support the needs of organizations in the e-Learning implementation, such as learning through the use of games. The experts claimed that the criteria of e-LS should cover such needs. The study also found that some vendor locked their software by providing particular features based on the license to users. The users were then tied to the software features provided by the vendor. When they wanted to add additional features, the users had to pay extra money. Moreover, if the number of users increased, they had to pay extra license fees. In some case, the organizations obtained unused software. The software comprised of bugs and provided poor performance.

\section{CONCLUSION}

This study reported the results from the investigation on the existing practices in the e-LS evaluation and selection. The results were gained from a survey conducted among experts from various organizations in Malaysia. The results covered the types of e-LS, the purpose of implementing e-LS, the approaches used in the implementation, evaluation and selection stages that were applied, the people involved in the evaluation, the evaluation techniques used, the evaluation criteria, the use of software tools, and the problems faced in the 
evaluation and selection process. Finding from this study show that there was no systematic guideline, consisting of a sequence of processes in the evaluation of e-LS. The study found that there were varieties of e-LS available in the market. Therefore, a comprehensive list of e-LS evaluation criteria is required in the evaluation of e-LS. These involve the quality aspects such as ISO/IEC 9126-1 quality model criteria and the non-technical aspects of e-LS. The e-LS evaluation criteria related to learning aspects should also be included to provide verities of evaluation criteria in the evaluation stages. The e-LS evaluation criteria and a suitable technique were required to evaluate e-LS. The experts from the study agree that a support tool needed to assist them specifically in the e-LS evaluation. This study is significant because it provides organization with preliminary information in the evaluation and selection of suitable e-LS for the e-Learning implementation. While the existing issues and problems identified in this study provide researchers with an opportunity to conduct further studies.

\section{REFERENCES}

[1] Adkins, S., 2014. The 2013-2018 AsiaSelfpaced eLearning Market Massive Demand for Self-paced eLearning Content in Asia.Middle East: Ambient Insight Research. http://www.ambientinsight.com/Resources/Docum ents/ AmbientInsight-2013-2018-Asia-Self-pacedeLearning-Market-Executive-Overview.pdf.

Accessed May 2017.

[2] Ali, A. 2004. Issues and challenges in implementing e-learning in Malaysia. http://asiapacific-odl2.oum.edu.my/C33/F80.pdf. Accessed 124 January 2017.

[3] Bersin J., Howard C., O’Leonard K., Mallon. D., 2009. Learning Management Systems. Bersin and Associates.

[4] Bhuasiri, W., Xaymoungkhoun, O., Zo, H., Rho, J. J., Ciganek, A. P., 2012. Critical success factors for e-learning in developing countries: A comparative analysis between ICT experts and faculty. Computers \& Education, 58, 843-855.

[5] Cavus, N., 2010. The evaluation of Learning Management Systems using an artificial intelligence fuzzy logic algorithm. Advances in Engineering Software, 41, 248 - 254.

[6] Chang, V., 2015. The role and effectiveness of elearning for the industry", https://eprints.soton.ac.uk/373732/. Accessed April 2017.

[7] Chang, V., 2016. Review and discussion: Elearning for academia and industry. International Journal of Information Management. 36, 476 485.
[8] Chua, B.B., Dyson, L.E. 2004. Applying the ISO 9126 model to the evaluation of an e-learning system. In Proc. of ASCILITE , 5-8.

[9] Colace, F., De Santo, M., Pietrosanto, A., 2006. Evaluation models for e-learning platform: an AHP approach. In Proceedings. Frontiers in Education. 36th Annual Conference. 1-6.

[10] Colace, F., De Santo, M., 2011. Evaluation models for e-learning platforms and the AHP approach: a case study. The IPSI BGD Transactions on Internet Research. 7, 1, 31 - 43.

[11] Choice, E, 2017 expertchoice ${ }^{\circledR}$, for Collaborative Decision Making. http://expertchoice.com.

Accessed January 2017.

[12] Dewal, S., Emmerich, W., Lichtinghagen, K. A decision support method for the selection of omss. In Systems Integration, 1992. ICSI'92, Proceedings of the Second International Conference on, 15 June, 32-40 (1992).

[13] Docebo, 2016. e-Learning Market Trends and Forecast 2017-202. https://eclass.teicrete.gr/modules/document/file.ph p/TP271/Additional\%20material/doceboelearning-trends-report-2017.pdf.

[14] Drigas, A.S., Tagoulis, A., Vrettaros, J, 2006. Development of asynchronous e-learning systems with the use of Java technology. In 2006 2nd International Conference on Information and Communication Technologies, 24 April, 1, 36-41.

[15] Esposito, F., Licchelli, O., Semeraro, G., 2004. Discovering student models in e-learning systems. Journal of Universal Computer Science. $10,47-57$.

[16] Floriana, O., Giovanni., 2004. Discovering student models in e-learning systemll. Journal of universal computer science. 10, 1, 47-57.

[17] García, F.B., Jorge, A.H., 2006. Evaluating elearning platforms through SCORM specifications. In IADIS Virtual Multi Conference on Computer Science and Information Systems (MCCSIS 2006), IADIS.

[18] Hussain, R. M. R.. 2004. E-learning in higher education institutions in Malaysia. International, E-Mentor. 7.

[19] Jadhav, A.S., Sonar, R.M., 2009. Evaluating and selecting software packages: A review. Information and software technology, 51, 555563.

[20] Jadhav, A.S., Sonar, R.M., 2011. Framework for evaluation and selection of the software packages: A hybrid knowledge based system approach, Journal of Systems and Software, 84, 1394-1407.

[21] Lai, V.S., Trueblood, R.P., Wong, B.K., 1999. Software selection: a case study of the application of the analytical hierarchical process 
to the selection of a multimedia authoring system. Information and Management. 36, 221-232.

[22] Nagi, K., 2006. Solving ethical issues in elearning. Special issue of the International Journal of the Computer, the Internet and Management. 14, 6.

[23] Nagi K., Suesawaluk Pp, U-Lan PV. 2008. Evaluating of eLearning Resource in A Learning management System (LMS) - A case study of Moodle and Open Source Platform. Special Issue of International Journal of the Computer. The Internet and Management. 16, 32.1 - 32.6.

[24] Padayachee, I., Kotze, P., \& van Der Merwe, A., 2010. ISO 9126 external systems quality characteristics, sub-characteristics and domain specific criteria for evaluating e-Learning systems. The Southern African Computer Lecturers' Association, University of Pretoria, South Africa.

[25] Rea, L. M., \& Parker, R. A., 2014. Designing and conducting survey research: A comprehensive guide. John Wiley \& Sons.

[26] Sarkis, J., Talluri, S., 2004. Evaluating and selecting e-commerce software and communication systems for a supply chain. European journal of operational research. 159, 318-329.

[27] Shee, D.Y., Wang, Y.S., 2006. Constructing a multi-criteria framework for evaluating asynchronous e-learning systems: a user satisfaction perspective. In International Conference on Information Society (i-Society 2006), Miami, FL, 10-July, 2006.

[28] Shee, D.Y., Wang, Y.S. 2008. Multi-criteria evaluation of the web-based e-learning system: A methodology based on learner satisfaction and its applications. Computers and Education. 50, 894 - 905.

[29] Teltumbde, A., 2000. A framework for evaluating ERP projects. International Journal of Production Research. 38, 4507- 4520.

[30] Thangaratinam, S., Redman, C.W.2005. The delphi technique. The obstetrician and gynaecologist. 7, 120 - 125.

[31] ISO/IEC 9126-1., 2001. Software EngineeringProduct Quality-Part 1: Quality Model, Geneva, Switzerland. International Organization for Standardization, 27.

[32] Kontio, J., 1996. A case study in applying a systematic method for COTS selection. In Software Engineering, 1996, Proceedings of the 18th International Conference on, 25 March. 201 $-209$.

[33] Wei, C.C., Chien, C.F., Wang, M.J.J., 2005. An AHP-based approach to ERP system selection. International Journal of Production Economics. 96, $47-62$. 